\title{
Comentario a la sentencia de inconstitucionalidad de la tabla de factores de ISAPRES: un aparente triunfo de los derechos sociales ${ }^{1}$
}

Este comentario, junto a los documentos que analiza, está disponible en www.anuariocdh.uchile.cl

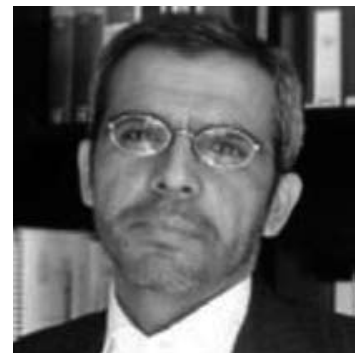

\section{Luis Cordero Vega}

Doctor en Derecho (U. de Lleida) y Magíster en Políticas Públicas con mención en Economía (U. de Chile). Profesor de Derecho Administrativo e investigador del Centro de Regulación y Competencia (RegCom), Facultad de Derecho, Universidad de Chile.

Icordero@derecho.uchile.cl

\section{RESUMEN}

El presente trabajo tiene por objeto explicar los alcances de la discusión que existió en torno a la dictación de la sentencia de inconstitucionalidad de la denominada "tabla de factores" de ISAPRES, al eliminar la posibilidad de discriminar administrativamente en los contratos privados de salud en razón de sexo y edad. Se explican los aspectos de la discusión y se precisa la manera en que el Tribunal decidió el caso, generando efectos en la operación del sistema de seguros de salud privado, pero indirectamente también en el público.

\section{Antecedentes}

En agosto de 2010 el Tribunal Constitucional (TC) ${ }^{2}$, actuando de oficio ${ }^{3}$, declaró la inconstitucionalidad del artículo 38 ter de la Ley de ISAPRES ${ }^{4}$, el cual regula el modo y las reglas que permiten determinar el precio de los planes de salud de las personas afiliadas las Instituciones de Salud Previsional (ISAPRES) ${ }^{5}$. Esta es la cuarta oportunidad en que el TC ejerce su potestad ${ }^{6}$ de

1 El objetivo del presente trabajo es comentar una sentencia y exponer una idea. Dada su extensión no corresponde a un trabajo dogmático. Agradezco las sugerencias formuladas a un texto inicial por parte de los profesores José Francisco García y Benjamín Mordoj. Por supuesto que los errores cometidos son de responsabilidad del autor. Se agradecen comentarios a Icordero@derecho.uchile.cl

2 STC Rol No 1.710, de 06 de agosto de 2010.

3 El proceso se inició de oficio luego de cuatro sentencias en las cuales declaró la inaplicabilidad de la disposición. STC Roles Nos 976, 1218, 1273 y 1287.

4 Ley $N^{\circ}$ 18.933, que correspondía al momento de su discusión al artículo 199 del DFL Nº 1, de 2005, del Ministerio de Salud.

5 El sistema chileno de ISAPRES descansa entonces sobre un supuesto de precios semidirigidos, donde se acepta algún grado de libertad en su determinación por parte de los oferentes, pero con un cierto margen de constreñimiento por la autoridad.

6 Los tres casos previos son: (a) artículo 171 del Código Sanitario (Solve et repete), STC Rol 1345, de 25 de mayo de 2009; (b) artículo 595 del Código Orgánico de Tribunales (gratuidad de turnos de abogados), STC Rol № 1254 , 
declarar la inconstitucionalidad de una norma legal y por esa vía expulsarla, con efectos generales, del ordenamiento jurídico. El uso de esta facultad, según explico infra, constituye el ejercicio más intenso de las potestades contramayoritarias reconocidas en nuestro texto constitucional.

El artículo 38 ter de la Ley de ISAPRES dispone que corresponde a la Superintendencia de Salud -órgano de la administración del Estado- establecer, mediante instrucciones generales, una "tabla de factores" que considera, entre otros elementos, el sexo y la edad de el o la cotizante, para calcular el riesgo de enfermedades de cada persona y de esta forma asignar precios a los planes de salud. La norma establecía, además, restricciones de intervalos de edad sobre los cuales debían descansar las competencias de la autoridad para fijar la tabla, de manera que las ISAPRES no disponen de total libertad para definir los precios de los contratos de salud ${ }^{7}$.

Desde que en el año 2007 se acogió un recurso de protección en contra de la modificación unilateral del plan de salud de una cotizante ${ }^{8}$, y por esta vía se solicitó la inaplicabilidad del artículo 38 ter de la Ley de ISAPRES ${ }^{9}$, comenzó a presentarse un importantísimo número de acciones en contra de las alzas en los precios de los planes ${ }^{10}$. De hecho, el número de acciones presentadas por este concepto constituyó el principal ingreso de causas en las Cortes de Apelaciones del país durante el año $2010^{11}$.

29 de julio de 2009; (c) artículo 116 del Código Tributario (delegación del Director Regional del SIl como juez tributario), STC Rol № 681, 26 de marzo de 2007.

7 En sus primeros tres incisos establece que: "para determinar el precio que el afiliado deberá pagar a la Institución de Salud Previsional por el plan de salud, la Institución deberá aplicar a los precios base que resulten de lo dispuesto en el artículo precedente, el o los factores que correspondan a cada beneficiario, de acuerdo a la respectiva tabla de factores. La Superintendencia fijará, mediante instrucciones de general aplicación, la estructura de las tablas de factores, estableciendo los tipos de beneficiarios, según sexo y condición de cotizante o carga, y los rangos de edad que se deban utilizar. Cada rango de edad que fije la Superintendencia en las instrucciones señaladas en el inciso precedente se sujetará a las siguientes reglas: 1.- El primer tramo comenzará desde el nacimiento y se extenderá hasta menos de dos años de edad; 2.- Los siguientes tramos, desde los dos años de edad y hasta menos de ochenta años de edad, comprenderán un mínimo de tres años y un máximo de cinco años; 3.- La Superintendencia fijará, desde los ochenta años de edad, el o los tramos que correspondan; 4.- La Superintendencia deberá fijar, cada diez años, la relación máxima entre el factor más bajo y el más alto de cada tabla, diferenciada por sexo; 5.- En cada tramo, el factor que corresponda a una carga no podrá ser superior al factor que corresponda a un cotizante del mismo sexo." A su vez, el artículo $2^{\circ}$, letra n) de la ley define la "tabla de factores" como: "aquella tabla elaborada por la Institución de Salud Previsional cuyos factores muestran la relación de precios del plan de salud para cada grupo de personas, según edad, sexo y condición de cotizante o carga, con respecto a un grupo de referencia definido por la Superintendencia, en instrucciones de general aplicación, el cual asumirá el valor unitario. Esta tabla representa un mecanismo pactado de variación del precio del plan a lo largo del ciclo de vida, el que es conocido y aceptado por el afiliado o beneficiario al momento de suscribir el contrato o incorporarse a él, según corresponda, y que no podrá sufrir variaciones en tanto la persona permanezca adscrita a ese plan".

8 Silvia Peña con ING Salud S.A, Rol No 4972-2007, de la Corte de Apelaciones de Santiago. La recurrente sostuvo que al cumplir 60 años, su plan aumentó unilateralmente el precio del plan de salud, de manera que si no estaba de acuerdo con el aumento podía modificar su plan de salud (reduciendo los beneficios pactados originalmente) o bien poner fin al contrato.

9 STC Rol No 976, de 26 de Junio de 2008. Esta sentencia tuvo los votos en contra de los Ministros Juan Colombo, Raúl Bertelsen y Jorge Correa Sutil.

10 Esta gran cantidad de acciones han dado origen inicialmente a decisiones inconsistentes entre la vía judicial (Corte Suprema) y la constitucional (Tribunal Constitucional). Mientras la primera ha descansado en la figura contractual para resolver estos conflictos, el TC lo ha expresado a través de la figura de derechos sociales. Recientemente en el caso Carrasco Delgado con ISAPRE CONSALUD (Rol No 8.837-2010, de 28.01.2011), la Corte Suprema ha razonado sobre la base de los criterios establecidos por el TC.

11 Vid. El Mercurio Domingo 13 de febrero, 2011, pág. A3. Según el Informe de la Comisión Asesora Presidencial en Salud, diciembre de 2010, pág. 18, mientras el año 2007 el 32\% de los recursos de protección que se presentaban en la Corte de Apelaciones de Santiago eran contra las ISAPRES, el año 2008 aumentó al $75 \%$ y el 2009 al $83 \%$. El informe se encuentra disponible en línea: http://www.redsalud.gov.cl/portal/url/item/96c1350fbf1a856ce040 01011f015405.pdf 
La recurrente en el recién mencionado recurso de protección señalaba que la facultad de modificar unilateralmente el precio de su contrato de salud en base a la tabla de factores era inconstitucional, pues violaba sus derechos a la igualdad ante la ley; el acceso, elección y permanencia en un sistema de salud; y la propiedad sobre el contrato. Al acoger este recurso, la Corte de Apelaciones utilizó atrevidos argumentos que hasta ese momento había sido reacia en invocar. En su sentencia sostuvo que:

a) la Constitución reconoce derechos sociales, entre los cuales está el derecho a la protección de la salud;

b) que conforme con una interpretación útil de la Constitución Política de la República, el deber de protección de los derechos fundamentales no sólo era exigible al Estado, sino también a los privados. Particularmente, las ISAPRES estaban sujetas al estatuto constitucional de la función social de la propiedad ${ }^{12}$;

c) el derecho a la protección de la salud es una exigencia constitucional, por lo que no puede disponerse de éste por convenciones entre privados, en especial en el caso del contrato de salud pues tiene base constitucional, de manera que sus cláusulas deben interpretarse y ser llevadas a la práctica en armonía con las normas constitucionales;

d) como consecuencia de que la interpretación constitucional maximice el goce de los derechos, la aplicación de la tabla de factores es insostenible constitucionalmente porque impone una distinción en el trato que restringe el derecho a la protección de la salud, y en consecuencia, deviene en una discriminación arbitraria ${ }^{13}$.

Desde la dictación de esta sentencia, se presentaron otras acciones, de diversas características, en las cuales el TC reprodujo argumentos idénticos para resolver las solicitudes de inaplicabilidad ${ }^{14}$.

Es en este contexto en el que el TC inició de oficio el procedimiento para revisar la constitucionalidad del artículo 38 ter de la Ley de ISAPRES.

\section{2. ¿Por qué la sentencia generó tanta expectativa?}

Existen seis razones que explican la importancia de lo decidido en esta sentencia y, por tanto, las expectativas que creaba.

12 La tesis del tribunal es que si la Constitución señala en el artículo 19 № 24 que "Sólo la ley puede establecer el modo de adquirir la propiedad, de usar, gozar y disponer de ella y las limitaciones y obligaciones que deriven de su función social. Esta comprende cuanto exijan los intereses generales de la Nación, la seguridad nacional, la utilidad y la salubridad públicas y la conservación del patrimonio ambiental", entonces es legítimo entender que los privados están sujetos a obligaciones en el ejercicio de su derecho de propiedad.

13 La tesis de la Superintendencia era que la precitada norma legal no era inconstitucional porque era el establecimiento del pago de una prima por riesgo, construida sobre la base de criterios estadísticamente significativos y, además, en el contexto de que los criterios sobre los cuales descansaba la tabla estaban acotados y reglados para la autoridad administrativa, de manera que se ajustaban plenamente a la Constitución. Por su parte, la ISAPRE sostuvo que no existía inconstitucionalidad, por las razones mencionadas por la Superintendencia, pero agregó además que la Constitución no garantizaba un sistema de salud indeterminado, sino la elección de uno, y que dado que este es un seguro privado de salud, el precio del plan era parte de ese sistema.

14 En la STC Rol No 1218, de 07 de julio de 2009, el TC se pronunció en el contexto de una solicitud de arbitraje ante la Intendenta de Fondos y Seguros de Salud, solicitado por María Osorio Sagredo con ISAPRE Cruz Blanca; en la STC Rol No 1273, de 20 de abril de 2010, el TC se pronunció a consecuencia del recurso de protección "Valenzuela Márquez con ISAPRE Banmédica", causa Rol No 9.451-2008, ante la Corte de Apelaciones de Santiago; STC Rol No 1287, 08 de septiembre de 2009; el TC se pronunció a consecuencia de la apelación de la sentencia en recurso de proyección caratulado Fernández Bitterlich con ISAPRE Golden Cross", causa Rol N 7779-2008, apelación ante la Corte Suprema. 
(a) La ley que había incorporado la tabla de factores tuvo por objeto limitar la discrecionalidad en los ajustes de precios de los planes, de manera de sustraer esta materia de la mera autonomía de la voluntad y de la autorregulación de las ISAPRES;

(b) En el mercado de los seguros de salud se presentan complejos problemas de asimetrías de información. Por lo tanto, estructuralmente hay condiciones concurrentes en los sujetos que hace que los precios de cada seguro deban ser diferentes, con la finalidad de acotar la selección adversa y el riesgo moral ${ }^{15}$ que normalmente tiende a una subprovisión del servicio ${ }^{16}$. Se trata, en suma, de un mercado con fallas intrínsecas;

(c) Es una circunstancia conocida que los costos sanitarios públicos tienden a aumentar, por razones tales como la esperanza de vida y la mejora en el acceso a medicamentos, de modo que cuando alguien "no paga la cuenta" esos costos terminan siendo trasladados; es decir, hay otras personas que sufrirán la menor disponibilidad de los recursos;

(d) Lo que estaba en juego era la manera como un sistema democrático acepta o no, frente a la evidencia estadística, que admitamos la diferenciación por sexo y edad. En otros términos, era el enfrentamiento entre una dimensión de la política pública con sesgos utilitaristas -tener recursos para prestaciones sanitarias de más personas, y una dimensión del sistema de derechos aparentemente igualitarista-, garantizar la condición de acceso al sistema sanitario a quien lo invoca.

(e) El Tribunal debía determinar los alcances y efectos de su declaratoria de inconstitucionalidad, considerando, especialmente, la gran cantidad de casos cuya resolución se encontraba pendiente tanto en las Cortes como en el propio TC.

(f) Esta discusión nos llevaría, en un futuro cercano, a debatir cómo debemos enfrentar la provisión privada de prestaciones de los servicios sanitarios desde la perspectiva de la cobertura de las políticas públicas.

Desde la perspectiva del Derecho público, todos y cada uno de estos elementos hacían y hacen esta discusión atractiva.

\section{La discusión en el Tribunal Constitucional}

El Tribunal abrió una etapa de audiencias públicas en las cuales todas las personas interesadas en la discusión podían formular sus observaciones y aportar sus antecedentes ${ }^{17}$. El Tribunal recibió las presentaciones del Poder Ejecutivo y de las ISAPRES ${ }^{18}$-las que reforzaron parte de los

15 En general, estas circunstancias se dan en el contexto de asimetrías de información (los que participan en la transacción no tienen la misma información disponible). Los efectos dependerán del momento en que se produce: estamos frente a un problema de riesgo moral cuando la asimetría ocurre después de la firma del contrato. Por ejemplo, la persona tiene incentivos al descuidado después de haber contratado un seguro de salud. Estamos frente a un problema de selección adversa cuando la asimetría ocurre antes de realizar la transacción: la compañía de seguro de vida no sabe si la persona que está al frente tiene buena o mala salud.

16 STIGLITZ, Joseph. Economía del Sector Público. Antoni Bosch editor, 2000, p.355. Este autor resume las críticas habituales al modelo de seguros médicos: “(a) algunas personas compran demasiado seguro y éste lleva a gastar excesivamente en asistencia sanitaria; (b) muchas personas no pueden conseguir un seguro (hay demasiada poca cobertura) o sólo pueden conseguirlo pagando unas primas excesivas; (c) los costos de transacción son excesivos".

17 El expediente público, donde constan las presentaciones de los interesados, es posible de revisar en: http://www. tribunalconstitucional.cl/index.php/rols/view/583.

18 Asociación de ISAPRES y las ISAPRES CONSALUD, BANMÉDICA, Vida Tres, Cruz Blanca, Colmena Golden Cross y Más Vida. 
argumentos invocados por éste-, además de personas de diferentes entidades que promovieron la declaratoria de inconstitucionalidad ${ }^{19}$.

En relación a los aspectos sustantivos del caso, el Poder Ejecutivo, en síntesis, defendió la constitucionalidad del artículo 38 ter de la Ley de ISAPRES. Tal diseño considera la provisión privada de un bien -seguro de salud- para quien pueda pagarlo, sin importar su precio, como un marco adecuado de política para el sector. El Ejecutivo defendió abiertamente el modelo de discriminación de precios utilizando la tabla de factores. Al efecto, señaló que: (a) el objetivo de la ley que creó la tabla de factores era evitar que las ISAPRES pudieran establecer libremente planes de salud que castigarán a los "afiliados cautivos" 20 ; (b) que la potestad de la Superintendencia de establecer la tabla de precios era parte de su competencia y que, por lo demás, evitaba la "petrificación" de los precios que podría producir la ley; (c) el plan de salud es un contrato de seguro que requiere de un sistema de ajuste de precios ya que "no cuenta con subvenciones estatales y/o mecanismos de ajustes de riesgos en salud"; (d) la tabla de factores se construye sobre la base de la evidencia estadística de "diferencias de gasto en salud por sexo y edad"; (e) observadas así estas variables, la diferencia corresponde a una "lógica sanitaria de base objetiva", cuyos elementos configuradores resultaban razonables y, en consecuencia, no eran violatorios de la igualdad constitucional; (f) el sexo y la edad son factores que normal y regularmente son utilizados en materia de salud por la literatura científica y también por las campañas del sistema público; (g) en la medida que las políticas de salud son focalizadas a grupos de la población, mejores serían sus resultados, por lo que la declaración de inconstitucionalidad podía tener efectos perniciosos en esa focalización; (h) la Constitución no garantiza un acceso ilimitado a las prestaciones de salud, sino tan sólo "un nivel mínimo "derivado de la integridad física y psíquica de las personas. Lo que la Constitución garantiza es la posibilidad de "elegir el sistema de salud al que deseen acogerse, pero dicha libertad no presupone el derecho a permanecer en un determinado plan sin asumir el mayor costo asociado a las prestaciones". A juicio del Ejecutivo, esta libertad de elección, pero no de permanencia, es una manifestación del principio de la subsidiariedad del Estado.

\section{La decisión del Tribunal}

El Tribunal declaró la inconstitucionalidad del precepto objetado sobre la base de una serie de consideraciones y prevenciones.

La sentencia comienza exponiendo la importancia y naturaleza de la inconstitucionalidad. Para estos efectos, el TC analizó la finalidad de la declaración de inconstitucionalidad de una norma y revisó sus efectos en el Derecho Comparado y en nuestro modelo constitucional ${ }^{21}$. Probablemente el Tribunal consideró necesario referirse a este tema para responder las objeciones de quienes lo exhortaban a no declarar la inconstitucionalidad argumentando que las consecuencias de la sentencia excedían su competencia para realizar un "examen de constitucionalidad abstracto", pues lo que se encontraba en juego era la aplicación de un sistema de cobertura de seguros de

19 Sociedad Javier de Fuenzalida y Compañía; Infolex Limitada; Corporación Humanas; Sociedad de Geriatría y Gerontología de Chile; los Diputados del Partido Socialista, señora Pacheco y señores Aguiló, Andrade, Castro De Urresti, Díaz, Espinoza, Lemus, Monsalve, Montes y Schilling; los profesores de Derecho Constitucional, señores Pablo Contreras, Gonzalo García, Tomás Jordán y Álvaro Villanueva.

20 Se considera "afiliados cautivos" a aquellas personas que teniendo planes de salud privados son calificadas como "riesgosas" por su edad, patologías de salud o sexo. Dichas personas para mantenerse en el sistema de salud privado deben conservar sus planes de salud y la ISAPRE a la que están afiliadas, pues elegir otro plan resulta mucho más caro y con una menor cobertura. Están en esa condición los adultos mayores y generalmente las mujeres en edad fértil.

21 Ver considerandos $1^{\circ}$ a $49^{\circ}$. 
salud y no una mera regulación general ${ }^{22}$. El Tribunal reafirmó sus facultades para realizar este tipo de análisis de constitucionalidad y procedió a realizar un juicio de ponderación conforme con el criterio de razonabilidad, teniendo presente sus dimensiones de adecuación o idoneidad, de necesidad y de proporcionalidad.

Luego, el Tribunal sometió el artículo 38 de la Ley de ISAPRES a este estándar de revisión y analizó si esta norma: (a) cumplía con ser adecuada a los fines constitucionales de tutelar la igualdad ante la ley, especialmente entre hombres y mujeres, proteger la salud de las personas incorporadas al sistema privado de las ISAPRES, particularmente en lo concerniente al rol preferente del Estado en garantizar la ejecución de las acciones de salud y proteger el libre e igualitario acceso a ellas para todas las personas; asegurar que las personas accedan al goce de las prestaciones básicas uniformes de seguridad social, garantizadas por la acción del Estado; (b) si era indispensable para alcanzar los fines señalados y (c) si las medidas guardaban proporcionalidad con tales objetivos ${ }^{23}$.

La respuesta del Tribunal, en votación dividida, fue que la norma señalada era incompatible con el derecho a la igualdad ante la ley, especialmente entre hombres y mujeres, y lesionaba, asimismo, el derecho a la protección de la salud y el derecho a la seguridad social ${ }^{24}$.

EI TC estableció la existencia de una violación al derecho a la igualdad ante la ley, pues no existía una fundamentación razonable para las distinciones en el trato en relación al sexo y la edad que permitía efectuar la norma objetada ${ }^{25}$, pero además porque tal norma afectaba lo que, en opinión del Tribunal, son las "igualdades esenciales" ${ }^{26}$ que no admiten tratos discriminatorios. Esto implicaba, además, que tampoco resultaba admisible -sin violentar la reserva de ley- en la regulación de derechos fundamentales que la Administración (Superintendencia) pudiera mediante meras "instrucciones generales" establecer discriminaciones ${ }^{27}$.

La sentencia también establece la existencia de una violación del derecho a la salud, pues en opinión del TC las normas objetadas infringían la Constitución al deslegalizar materias básicas de la seguridad social y desatender el deber del Estado de garantizar el acceso a la salud ${ }^{28}$. En su razonamiento, las formas y condiciones de la ejecución de las acciones de salud debían determinarse por la ley con un grado de precisión tal que impidiera que el ejercicio de la discrecionalidad administrativa se extendiera a la vulneración de los derechos constitucionales, como lo permitiría la determinación de la tabla de factores por vía administrativa ${ }^{29}$.

EI TC volvió a invocar el razonamiento que había aplicado en otras solicitudes de inaplicabilidad al sostener que el contrato de salud no estaba sujeto a la libertad contractual, sino que debía enmarcarse en las reglas del orden público, pues buscaban regular aspectos propios de un derecho

22 Ver MONTT, Santiago. "Sistema de ISAPRES y Constitución", en A2 El Mercurio, 07 junio de 2010.

23 Ver considerando 142.

24 Ver considerando 144.

25 Ver considerando 144.

26 El estándar de igualdades esenciales como complemento de la "igualdad ante la ley" fue desarrollado por el TC en la STC Rol No 1273. En esta sentencia el TC sostuvo que la diferenciación por sexo y edad que permite el artículo 38 ter de la Ley 18.933, establece un trato desigual para igualdades esenciales, como son: (i) la igualdad en dignidad y derechos con que las personas nacen (inciso primero del artículo 1 de la Constitución), (ii) la igualdad de oportunidades como derecho de las personas en la participación en la vida nacional (inciso quinto del artículo 1 de la Ley Fundamental), (iii) la igualdad ante la ley entre el hombre y la mujer (oración final del inciso primero del número 2 del artículo 19 de la Constitución), y (iv) la igualdad de acceso a las acciones de salud (inciso segundo del número 9 del artículo 19 de la Constitución) (C. 148).

27 Ver considerando 145.

28 Ver considerandos 150 y 151.

29 Ver considerando 158. 
constitucional $^{30}$. Igualmente, indicó que el contrato de salud estaba destinado a garantizar el acceso a las prestaciones salud, de modo que precios desproporcionados en relación a las rentas, determinados sobre la base a factores como el sexo y la edad, ambos inherentes a la condición humana, afectaban el libre e igualitario acceso a las acciones de salud que el Estado está obligado a garantizar ${ }^{31}$. Nótese cómo el TC implícitamente entendió que la introducción de mayor competencia al sector, de modo consistente con las potestades en materia de libre competencia vigentes en la propia Constitución, no fue considerada como una herramienta suficiente para resguardar tales objetivos de orden público, desde la perspectiva de la disipación de rentas extraordinarias que se presumen como consecuencia de precios desproporcionados, es decir, la generación de competencia entre planes para evitar ingresos sobrenormales de las ISAPRES.

Las consideraciones anteriores condujeron al TC a afirmar que también se lesionaba el derecho a la seguridad social. En la sentencia se indica que al no establecerse en forma fija la relación máxima posible entre el más alto y el más bajo de los costos asociados a los factores de cada "tabla de factores" y ninguna precisión ni regla especial para las personas que se encontraban en situación de obtener las prestaciones de seguridad social ${ }^{32}$, se vulneraba el propósito esencial de la afiliación de las personas al sistema privado de ISAPRES. Dicho propósito consistía en "la necesidad de proteger de estas contingencias [de salud] al ser humano y a los que de él dependen emana de su derecho a la existencia; de la obligación de conservar su vida y hacerlo en un nivel digno y acorde con su condición de tal"33.

La tesis del TC en este punto es que la norma objetada era contraria a los principios de solidaridad y de equidad que informaban, en su opinión, no sólo la seguridad social, sino todo el conjunto de derechos fundamentales sociales garantizados en la Constitución ${ }^{34}$.

Finalmente, el TC hizo varios alcances a los efectos de su declaratoria de inconstitucionalidad, lo que tiene importancia general en las decisiones de este órgano. Sostuvo que: (a) tal como lo señala la Constitución, la declaratoria de inconstitucionalidad no tiene efectos retroactivos y produce sus efectos desde que se publica la sentencia en el Diario Oficial ${ }^{35}$; (b) no obstante, considerando que los contratos de salud son de tracto sucesivo y sus normas son de orden público, esto no significaba que el contenido de los contratos suscritos con anterioridad no puedan verse afectados por esta declaración del Tribunal ${ }^{36}$; (c) que la sentencia no afectaba la resolución de las inaplicabilidades en tramitación ${ }^{37}$; (d) que la expulsión de una ley por declaratoria de inconstitucionalidad es una "derogación sui generis", pues deja sin efecto una norma legal no por razones de mérito, sino porque ella posee un vicio incompatible con la Constitución, dado que la declaración de inconstitucionalidad es analizada como si fuera "un vicio sobreviniente", de manera que eso explica la imposibilidad de aplicación retroactiva como establece la Constitución ${ }^{38}$.

30 Ver considerando 154.

31 Ver considerando 155. La opinión del TC en esta parte fue que "para ajustarse a la garantía constitucional reseñada, el legislador debió establecer parámetros prudentes, dentro de latitudes razonables, al determinar las condiciones a las que debe ajustarse la fijación del precio de un seguro de salud que se contrate con una ISAPRE".

32 Ver nota 7.

33 Ver considerando 159.

34 Ver considerando 161.

35 Ver considerandos 166 y 167.

36 Ver considerando 170.

37 Ver considerandos 164 y 165.

38 Ver considerando 171. 


\section{Las implicancias y los dilemas aún en discusión}

Desde la perspectiva del Derecho público y sus efectos en las políticas públicas, son muchas las consecuencias de esta sentencia, pero por ahora sólo me detendré en las más evidentes.

Como he sostenido a otro apartado, el rol del Tribunal Constitucional es complejo, porque es una institución contramayoritaria (resguarda ciertas reglas a pesar de las mayorías), que interpretando la Constitución (sin posibilidad de revisión posterior) define: (a) los límites de la convivencia posible; (b) el respeto por las minorías (de todo tipo, incluidas las políticas); (c) la contención en el momento preciso en que la política desborda al derecho. En resumen, la pregunta, aparentemente simple para una democracia de qué Constitución nos rige, tiene una respuesta compleja: la que defina el Tribunal Constitucional mediante su interpretación ${ }^{39}$.

Cuando el TC señala que su objetivo no sólo es garantizar el texto escrito de la Constitución, sino también "sus valores y principios", entonces es un tribunal que puede ser deliberante de la política pública o, en último término, un censor moral. Ese es el riesgo inmanente a ese argumento ${ }^{40}$, pues permite a un órgano, no democrático en su origen y de más fácil captura que aquellos configurados en el marco de procesos políticos ${ }^{41}$, definir con fuerza de autoridad nuestra concepción sobre aquello que es moralmente admisible.

Afirmar que en este caso el TC no deliberó sobre la manera de entender la política pública sanitaria es ingenuo, por más esfuerzos que realice el propio Tribunal de asilarse en la "declaración abstracta de inconstitucionalidad". A mayor abundamiento, esta situación resulta clara si se considera que el TC desarrolló una teoría de derechos sociales que hasta ahora nuestra jurisprudencia y el propio constituyente derivado de 2005 habían sido reacios en aceptar ${ }^{42}$.

Pese a mi preferencia personal, no puedo dejar de advertir que todo el razonamiento en esta materia está hecho sólo para los contratos de seguros de salud, lo que hace que su análisis sea parcial y, en consecuencia, limitado desde la dimensión social que el Tribunal pretende construir desde la "solidaridad". En efecto, si el razonamiento del TC es consistente, entonces no es admisible la reserva presupuestaria y el modelo de atención del sistema público. En el caso "ISAPRE" el TC dijo expresamente que:

"El aumento de los precios al amparo de la norma cuestionada puede traer consigo, y así ocurre en la realidad de muchas personas, una completa imposibilidad de costearlos, ocasionando la indignidad que ello significa para el trascurrir de sus vidas y la evidencia de la falta de

39 CORDERO VEGA, Luis. Las contiendas de competencia en el Tribunal Constitucional tras la reforma constitucional de 2005. Revista de Derecho Público (72); 2010. Departamento de Derecho Público, Facultad de Derecho, Universidad de Chile, Santiago, Chile.

40 Vid. CORREA SUTIL, Jorge. Inaplicabilidad por inconstitucionalidad en la Jurisprudencia del Tribunal Constitucional. Abeledo Perrot-Legal Publishing. Santiago, 2011, p. 41.

41 GARCíA, José Francisco. El principio de proporcionalidad como parámetro de control de la legislación de interés especial. Anuario de Derecho Público (1); 2010, UDP, p. 448, nota 54, citando a Elhauge (Does Interest Group Theory More Intrusive Judicial Review. Yale Law Journal (101): 31-75), señala que los grupos de interés podrían no ser tan influyentes en el proceso político y que las propuestas para expandir el control judicial sufren la falacia de nirvana, ya que el proceso de adjudicación, como el de regulación, son imperfectos y susceptibles de influencia de los grupos de interés.

42 Basta recordar que, como consta de la historia fidedigna de la ley de reforma constitucional No 20.050 del año 2005, se descartó que la Constitución se refiriera al Estado social, dejando esta definición, dadas las preferencias existentes, "a cargo del devenir político constitucional de nuestro tiempo". Ver Historia de la Ley № 20.050, (Biblioteca del Congreso Nacional, 2005) 1051-1054. El Tribunal en sus STC Roles No 976 (c.29), 1218 (c.23) y 1287 (c.23), sostuvo que "la satisfacción de tal exigencia [protección a la salud y la seguridad social] representa un rasgo distintivo de la legitimidad sustantiva del Estado Social en la democracia constitucional contemporánea". 
concreción de la obligación que el texto de la Constitución le asigna al Estado para dirigir su acción a garantizar el acceso de todos los habitantes al goce de prestaciones básicas uniformes" ${ }^{43}$.

No obstante, la consecuencia de política pública del encarecimiento de los planes de salud será el problema obvio de todo sistema sanitario. Los que carezcan de recursos utilizarán el sistema público, sistema que se congestionará y afectará precisamente a quienes no pueden elegir un modelo de seguro, porque no están en condiciones ni han estado nunca en condiciones de costearlo ${ }^{44}$. Sin embargo, el TC parece no ver que aquello sometido a su conocimiento y decisión fue, esencialmente, la discusión acerca de qué diseño de política sanitaria en materia de cobertura de salud es más consistente con un Estado democrático ${ }^{45}$. Simplemente presumió su existencia y legitimidad.

La ironía de todo esto, es que interpretar la Constitución ajustándose a sus valores y principios para proteger un tipo de acceso a la salud, puede tener un efecto regresivo significativo. Particularmente, para quienes no estando en la discusión de un contrato de salud privado. Quienes reciben cotidianamente prestaciones de un congestionado servicio público, carecen en su gran mayoría de recursos para elegir un seguro de salud privado. A menos que la política pública se oriente hacia un sistema de financiamiento solidario entre ambos sistemas, la sentencia del TC mantiene la profunda inequidad en las prestaciones que reciben los usuarios públicos ${ }^{46}$.

Además del efecto regresivo recién mencionado, el TC nuevamente utilizó la tradicional concepción de la reserva de ley. Dicha invocación constituye una manifiesta sospecha hacia las decisiones de las autoridades administrativas. Tras esta decisión, lo que se olvida es que para el logro efectivo del sistema de derechos sociales es indispensable la intervención y la libertad de las agencias administrativas. El criterio del TC, con la finalidad de sostener su declaratoria de inconstitucionalidad, reivindica un estándar que limita fuertemente el funcionamiento, precisamente, del Estado Social.

43 Considerando 160.

44 Según los datos de FONASA, esta institución del Estado atiende a 12,5 millones de personas que son sus beneficiarios, que corresponden al 74\% de la población. Quienes cotizan en FONASA son sólo 4,9 millones de personas. En cambio las ISAPRES cuentan con 2,8 millones de beneficiarios de los cuales 1,4 son cotizantes. Ver Informe n. 11, pp. 16 y 17.

45 En tales circunstancias es sensato pedirle al TC un estándar de proporcionalidad básico, considerando los efectos generales que tiene en la política pública una declaratoria de inconstitucionalidad y teniendo en cuenta los efectos agregados que en los derechos de otras personas produce la decisión. En efecto, tras esto existe la necesidad de que quienes adjudican tengan claro que los recursos públicos son escasos y que la gestión pública debe proveer de garantías de dignidad común mínima a todas las personas y no sólo a quienes pueden acceder, comparativamente en condiciones de privilegio, a contratos privados de salud. Ver en este sentido, HOLMES, Stephen y SUNSTEIN, Cass. The Cost of Rights. Why Liberty Depends on Taxes. New York, W.W. Norton \& Company, 1999, p. 120.

46 Ver Informe de la Comisión Asesora Presidencial n. 11, p. 37. 